\title{
Supervised Learning for Enhanced Early HARQ Feedback Prediction in URLLC
}

\author{
Saleh AlMarshed \\ $5 G$ Innovation Centre (5GIC) \\ Institute for Communication Systems Institute for Communication Systems \\ University of Surrey, UK \\ s.almarshed@surrey.ac.uk
}

\author{
Klaus Moessner \\ Professorship for Communications Engineering \\ Technical University Chemnitz, Germany \\ and 5G Innovation Centre \\ Institute for Communication Systems \\ University of Surrey, UK \\ klaus.moessner@etit.tu-chemnitz.de
}

\begin{abstract}
The fifth-generation wireless communication networks (5G) facilitate a wide range of newly-emerging applications alongside existing cellular mobile broadband services. One of the key service classes of $5 \mathrm{G}$ is Ultra-Reliable and Low-Latency Communications (URLLC), which guarantees the rapid delivery of short packets (up to $1 \mathrm{~ms}$ ) with a success probability rate of $99.999 \%$. The challenging reliability and latency requirements of URLLC cannot be delivered by existing cellular networks, resulting in the need for significant air interface modifications. This study aims to satisfy the link latency requirements of URLLC applications, and specifically reduce the latency associated with the presence of the Hybrid Automatic Repeat reQuest (HARQ) feedback scheme. To this end, we investigate a supervised learning method to provide early HARQ (E-HARQ) feedback on the decodability status of the coded-received signal, ahead of the decoding processing. This strategy allows the transmitter to react faster and minimize the signal round-trip time (RTT). The simulation results demonstrate the capability of the proposed mechanism to speed up the feedback releasing and enhance the prediction accuracy by $12 \%$ with the introduction of a new feature derived by the channel state estimation.
\end{abstract}

Index Terms-5G, HARQ, Latency, Machine Learning, URLLC.

\section{INTRODUCTION}

Each new generation of cellular networks sets several optimistic goals in order to improve the network capacity and user data rate of previous generations. The fifth-generation wireless communication network $(5 \mathrm{G})$ goes beyond this by supporting use cases that have diverse ways of consuming the same network's resources. Plans include enabling interactive and high-performance applications to run alongside enhanced broadband services, using autonomous machines and devices, rather than the human-operated mobile terminals that were the primary consumers of traditional broadband services. UltraReliable and Low-Latency Communications (URLLC) is one of the vital service classes of $5 \mathrm{G}$ with the aim of supporting applications that depend on mission-critical links. Since the human reaction time can be within a range of tens of milliseconds, the packet transmission time for these mission-critical applications should be tens of hundreds of microseconds. However, these strict latency requirements cannot be met by the current Long-Term Evolution (LTE) networks that expe- rience a range of end-to-end latency between 30-100 ms [1]. This calls for significant optimization of the radio interface. A set of new proposals has been driven by both academia and industry to fulfill URLLC requirements. Among these promising solutions, we believe that the Hybrid Automatic Repeat reQuest (HARQ) procedure can be the bottleneck that needs to be optimized in order to achieve the strict latency requirements, where the latency is traded for spectral efficiency while maintaining the network reliability target. The HARQ process can be seen as a combination of forward error-correcting coding and error detection, both performed at the receiver, to release a negative-acknowledgment message (NACK) when a decoding failure due to poor channel conditions is experienced. The interval between sending the packet, followed by receiving the ACK/NACK from the receiver until the re-sending of the previous (or new) packet, is known as the round-trip time (RTT) [2]. Minimizing the duration of this is considered as the key to meeting the challenging URLLC latency requirement. Therefore, this is the motivation for our work, in which we design an HARQ estimator capable of delivering early feedback regarding the decodability status of a packet at an early stage (i.e., ahead of the completion of the full decoding process). The problem of the optimization of the HARQ mechanism has been addressed in the literature from diverse aspects. The authors in [3] proposed a classification algorithm to estimate the feedback, based on a feature inspired by the log-likelihood ratio (LLR) that is extracted following the demodulation of the received data. The authors in [2] aimed to further expedite the feedback by allowing the estimation decision to be made after extracting only a part of the codeword. The proposed approach employed low-density parity-check (LDPC), as this offers excellent opportunities to exploit substructures, while also being adopted by the $3^{\text {rd }}$ Generation Partnership Project (3GPP) as a channel coding scheme for enhanced mobile broadband (eMBB) in $5 \mathrm{G}$ networks [4]. Furthermore, a machine learning (ML) approach is utilized in [5] to enhance the early feedback prediction instead of the hard threshold-based method (HT). The same feature (i.e., LLRs) is used to train the ML model with a range of different classification algorithms, such as logistic regression 
and random forest. Although the E-HARQ feedback schemes (such as [2]-[5]) seem to achieve an interesting latency enhancement, these mechanisms depend on the channel decoding task to release the early feedback. We believe that associating the early feedback with the decoding process can be an obstacle to achieving the strict latency requirement of URLLC. Particularly when considering the significant delay caused by channel decoding task (i.e., consuming approximately $60 \%$ of the total processing time in LTE [6]), in this paper, we aim further to enhance the early feedback classifier through the following contributions:

- We enhance the classifier accuracy by introducing a new feature that concludes from channel state estimation. Simulation results demonstrate an accuracy enhancement of approximately $12 \%$ compared to other techniques in the relevant literature.

- Our proposed features-extraction mechanism does not rely on the channel decoding task; hence the feedback is released at an earlier stage, compared to existing proposals.

- We train the ML-based classifier with a standardized dataset that is collected from a standard-compliant 3GPP 5G New Radio (NR) Release 15 waveform.

The remainder of the paper is organized as follows: in Section II the early HARQ feedback strategy is discussed along with the definition of the user plane latency and the delay model. Section III describes the system model. Section IV presents the performance evaluation and simulation results, and finally, section $\mathrm{V}$ provides concluding remarks and plans for future work.

\section{EARLY HARQ FEEDBACK}

The physical layer retransmission HARQ is a well-known mechanism aiming to enhance the data transmission reliability at the cost of additional link latency. In traditional HARQ, the feedback is coupled with the channel decoding and error detection tasks, where the received payload is firstly decoded, followed by applying the Cyclic Redundancy Check (CRC) to check the integrity of the decoded bits. As a result, the HARQ feedback is released, either asking for further redundancy information (NACK) or acknowledging the success of the reception (ACK). Another approach is to enable early HARQ feedback, which can be realized by decoupling the channel decoding task from the feedback releasing. This mechanism aims to build a predictor ahead of the decoder in order to estimate the decodability outcome of the received coded block at an early stage. However, the early prediction may suffer from two potential forms of error. Firstly, when the feedback is estimated to be ACK for a codeword, but it is then recognized that the decision was falsely estimated (i.e., after the CRC task), resulting in a false positive decision; and secondly, when the feedback is predicted to be NACK for a codeword that could be successfully decoded, resulting in a false negative decision. Nevertheless, obtaining a reliable classifier can effectively mitigate the emerging of these forms of errors.

\section{A. Latency Analysis}

To evaluate the anticipated benefit of employing E-HARQ, we need to analyze the link latency carefully. Generally, the link latency can be defined differently at different layers of the communication protocol stack. In this study, we consider the user plane latency, which is defined as the time it takes to successfully deliver an application layer packet from the radio protocol layer $2 / 3$ service data unit (SDU) ingress point to the radio protocol layer 2/3 SDU egress point of the radio interface [7]. To gain a deep insight into the components that constitute the user plane delay, let find the total latency on the downlink direction when the receiver radio successfully decodes the packet from the first transmission. The total latency can be written as:

$$
T_{t}=T_{A G}+\triangle_{B S}+T T I_{T x}+2 \rho+\triangle_{U E}+T T I_{R x},
$$

where the frame alignment delay is denoted by $T_{A G}$, the payload transmission time is denoted by $T T I$, the propagation delay is denoted by $\rho$, and the processing times at the transmitter (i.e., the base station) and lastly the receiver (i.e., the user equipment) are represented by $\triangle_{B S}$ and $\triangle_{U E}$, respectively. The downlink transmission scenario is depicted in Fig. 1.

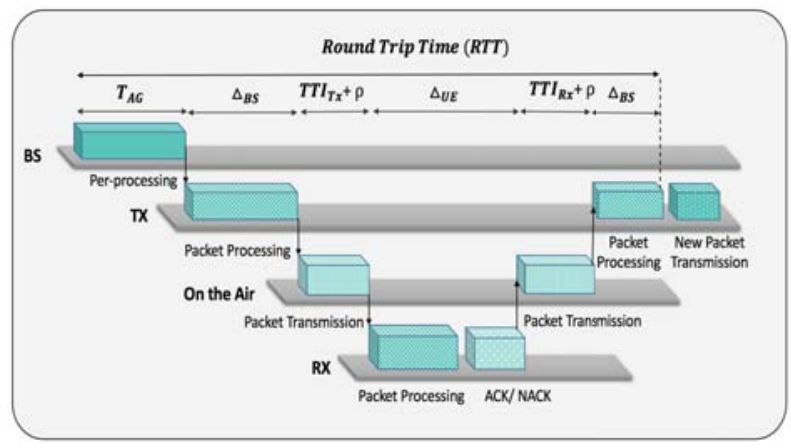

Fig. 1. User-plane latency model on the downlink.

Let us now consider a URLLC payload that travels on the downlink direction from the base station (BS) to the user equipment (UE). Each packet contains a total of $K$ symbols. The packet spans over the time domain for 7 Orthogonal frequency-division multiplexing (OFDM) symbols, within a duration of time, referred to as TTI. The period of every TTI mainly depends on each symbol's subcarrier spacing. Unlike the legacy LTE, 5G NR adopts multiple subcarrier spacing types (i.e., 15, 30, 60, and $120 \mathrm{kHz}$ ) that offer a higher degree of flexibility to satisfy the diverse requirements $5 \mathrm{G}$ use cases. It is worthwhile to mention that employing a wider subcarrier spacing leads to a shortening of the TTI duration, thus reducing the processing and buffering time. This strategy is considered one of the primary keys to realizing the strict latency requirements of URLLC. When the payload arrives from the core network, it can be acquired by the BS instantly in the $(0, \mathrm{TTI})$ interval. Following this, the transmitter will consume $\triangle_{B S}$ to process the signal and then stack it up alongside the other 'ready-to-fly' packets. This queue delay 
is part of the alignment delay $T_{A G}$ that is accounted from the instant of the packet arrival until the instant when the least significant bit was sent (i.e., a random variable mainly dependent on network load). After this, the packet will travel through the air-transmission medium, experiencing a propagation delay $\rho$ (i.e., affected mainly by the distance between the BS and the UE). Finally, the UE will consume $\triangle_{U E}$ to receive the packet and perform the baseband processing such as decoding, channel estimation, and signal detection. Table I summarizes the two-way latency components for different subcarrier spacing values. We assume that the processing time of $5 \mathrm{G} \mathrm{UE}$ is compliant with the 3GPP standard, known as processing capability 2 for low latency service [8]. On the other hand, the processing delay at the BS is assumed to be equal to the processing time at the UE, since the processing time of network elements is still not yet defined. It is essential to mention that the previous delay components solely cover the user-plane latency; there are still other delay sources between the devices, and the core network should also be taken into account to evaluate the overall link latency [9]. Let us now examining the expected reduction on the link latency after enabling the E-HARQ mechanism. As our proposal relies on releasing the feedback ahead of the channel decoding task, we expect a delay-saving equivalent to the decoding processing time. Since the channel coding method of URLLC is still not defined yet, we make a conservative assumption to estimate the decoding delay, which is derived by the turbo decoding delay on LTE, which is about $60 \%$ of the total UE's processing time [6]. Assuming that the packet is successfully delivered in the first round trip, the two-way link latency can be obtained as follows:

$T_{t}=T_{A G}+\triangle_{B S}+T T I_{T x, R x}+2 \rho+(1-0.6)\left(\triangle_{U E}\right)$.

Accordingly, The RTT can be obtained by rounding the twoway delay to the nearest multiple of TTI, as shown in Table II. It is essential to mention that the promising latency reduction was evaluated based on some LTE hardware constraints, we expect an extra latency reduction in case of any potential future improvements on the hardware of 5G NR. Also the expected reduction gain can be accumulated with each additional signal retransmission, in the case of failure of the first packet transmission.

TABLE I

TWO-WAY LATENCY WITH DIFFERENT SUBCARRIER SPACING

\begin{tabular}{lllll}
\hline Delay Component $\mathbf{( m s )}$ & Symbol & $\mathbf{1 5} \mathbf{~ k H z}$ & $\mathbf{3 0} \mathbf{~ k H z}$ & $\mathbf{6 0} \mathbf{~ k H z}$ \\
\hline TTI duration & $T_{T T I}$ & 0.5 & 0.25 & 0.125 \\
Frame Alignment & $T_{A G}$ & 0.25 & 0.125 & 0.625 \\
UE Processing & $\triangle_{R x}$ & 0.36 & 0.19 & 0.19 \\
BS Processing & $\triangle_{T x}$ & 0.36 & 0.19 & 0.19 \\
Propagation Delay & $\rho$ & 0.003 & 0.003 & 0.003 \\
\hline Total (one way) & & 1.47 & 0.76 & 0.57 \\
\hline Total (Two-way) & & 2.95 & 1.52 & 1.14 \\
\hline RTT (One HARQ Round) & & 3 & 1.5 & 1.25 \\
\hline
\end{tabular}

\section{SYSTEM MODEL}

Due to the novelty of applying ML in physical layer design and the fact that URLLC standardization activities are still in progress, there is no suitable public dataset available for our research. We therefore built (1) a Wireless communication link model (M1) to collect the training and validation dataset (i.e., the features and decodability status); and (2) a MLbased classifier (E-HARQ), which can be trained to predict the decodability of the received signal using the collected samples from (M1).

\section{A. Wireless Communication Link Model (M1)}

We modeled a wireless communication link to perform the operations of the transmitter, channel modeling, and receiver, and then to analyze the link performance by computing the Block Error Rate (BLER). In this model, we consider a point-to-point multiple-input multiple-output (MIMO) system, which has $N_{t} \cdot N_{r}$ transmitter and receiver antennas, respectively. A URLLC message (M) travels on the downlink shared channel (DSCH). The message consists of a number of bits $B$ that are encoded to a codeword $L$ via an LDPC encoder. Each bit on the codeword is mapped to a symbol using an appropriate modulation scheme (i.e., QPSK, 16 QAM). Then, the synchronization words are inserted into the modulated codeword $S$ while processing the other transmitter functions, such as MIMO precoding and resource mapping. Afterwards, the modulated codeword is transmitted over a wireless channel, assuming each symbol in the codeword has experienced the same channel fading effect. Hence, the received signal can be expressed as

$$
r=H S+w,
$$

where $S$ is the transmitted signal; $H \in C^{N_{t} N_{r}}$ is the fading matrix, that is assumed to support clustered delay line (CDL) [10]. Finally, $w$ denotes the additive white Gaussian noise (AWGN), which has independent $w \sim C N(0,1)$. The arrived signal is then processed by the well-known minimum meansquared error equalizer (MMES), using the channel information that is assumed to be known in this study. The equalized signal can be found as:

$$
r^{\prime}=r * H^{-1} .
$$

The first feature $B E R_{L}$ after that can be extracted by estimating the average bit error rate, ahead of the full decoding process. The extraction strategy initiate by finding the LLRs for each received bit at the codeword to being either 1 or 0 as expressed below:

$$
L\left(b_{k}\right)=\log \frac{P\left(b_{k}=1 \mid y\right)}{P\left(b_{k}=0 \mid y\right)},
$$

Following that, the demodulated signal is decoded using the belief propagation algorithm. Through the decoding process, the LLRs are collected after a few decoding iterations (i.e., 
TABLE II

ROUND TRIP TIME COMPARISON BETWEEN TRADITIONAL HARQ AND E-HARQ

\begin{tabular}{lcccccc}
\hline Sub-carrier Spacing & \multicolumn{2}{c}{$\mathbf{1 5} \mathbf{~ k H z}$} & \multicolumn{2}{c}{$\mathbf{3 0} \mathbf{~ k H z}$} & \multicolumn{2}{c}{$\mathbf{6 0} \mathbf{~ k H z}$} \\
\hline Early HARQ Mode & OFF & ON & OFF & ON & OFF & ON \\
Two-way latency (ms) & 1.47 & 1.26 & 1.52 & 1.29 & 1.14 & 0.91 \\
Round Trip Time (ms) & 3 & 2.5 & 1.5 & 1.25 & 1.25 & 1 \\
\hline Latency Reduction & & $\mathbf{1 6 . 7 \%}$ & & $\mathbf{1 6 . 7 \%}$ & & $\mathbf{2 0 \%}$ \\
\hline
\end{tabular}

partial decoding). Then, the probability of each bit being incorrectly estimated is calculated as follows [3]:

$$
P_{w}\left(b_{k}\right)=\frac{1}{1+\left|L\left(b_{k}\right)\right|},
$$

Finally, the feature can be extracted by finding the average error rate for each codeword as:

$$
B E R_{L}=\frac{1}{M} \sum \frac{1}{1+P_{w}} .
$$

The second Channel State Estimation (CSI) feature is derived by the channel state estimation. When the UE receives the transmitted signal affected by complex channel gain and noise, the known pilot symbols at both parties can be utilized to estimate the channel conditions and then equalize the channel's effects on the received signal. The instantaneous channel estimation for a subset of Resource Elements (REs) within a subframe can be computed using the least-squares estimate [11] as follows:

$$
H_{p}(k)=\frac{Y_{p}(k)}{X_{p}(k)},
$$

where $H_{p}(k)$ represents the channel response for the RE occupied by the pilot symbol, $Y_{p}(k)$ is the received pilot symbol, and $X_{p}(k)$ represents the known transmitted pilot symbol, the proposed feature is after that extracted by computing the average of estimated pilots within a codeword. Finally, The dataset labels (i.e., the decodability outcomes) are collected after the completion of the receiver tasks, including the process of checking the integrity of the decoded bits.

\section{B. Early HARQ Predictor}

On the conventional E-HARQ method (i.e., non-ML-based), the early prediction is achieved by mapping each extracted $B E R_{L}$ feature to an ACK or NACK message, based on a hard threshold that is experimentally obtained [2]. On the other hand, the ML-based model is trained to classify the collected features to deodability outcomes (i.e., one when the block is decodable, or 0 if it is not decodable). For this purpose, we nominate a binary classification method known as logistic regression (LR). It shows excellent generalization performance in the processing of high-dimensional data with the presence of many irrelevant features [12]. We note further that employing logistic regression for an E-HARQ classifier in [2] demonstrated good overall performance compared to other classification methods such as Random Forests and Isolation Forests. To build a reliable E-HARQ classifier, we initially train the model offline to find the optimum parameters that will return the minimum value of cost function, which is defined as the difference between the model output (predicted values) and the dataset output (actual values). Finally, the generalization ability for the model is evaluated using the validation dataset. The following section provides a brief system evaluation for the proposed E-HARQ.

\section{PERFORMANCE EVALUATION}

We compare the performance of the proposed E-HARQ mechanism with another existing algorithm in the literature that relies on the log-likelihood ratio to perform the classification. As the URLLC frame structure and coding schemes are still under standardization, we collect a reliable dataset from a standard-compliant waveform of 3GPP 5G NR Release 15 [13]. The simulation parameters are illustrated in Table III. In all cases, we vary the SNR values at low/moderate channel conditions for 500,000 blocks. This particular operational region characterized with two key features, Firstly, the significant gain from employing E-HARQ is realized when the user experiences poor channel conditions, whereas the probability of the transmission to be decoded in the first round is significantly higher in good channel conditions. Therefore, the E-HARQ relative gain will be less significant. Secondly, in these SNR regions, a balanced dataset can be obtaining where the dataset labels at each class are almost equal. In other words, the number of correct blocks is similar to the number of corrupted blocks; this can prevent the classifier from being biased to the class that has a major number of samples.

TABLE III

SYSTEM-LEVEL EVALUATION PARAMETERS

\begin{tabular}{l|l} 
Parameters & Description \\
\hline Waveform & MIMO, CP-OFDM \\
\hline Subcarrier Spacing & $30 \mathrm{KHz}$ \\
\hline Bandwidth & $20 \mathrm{MHz}$ \\
\hline Modulation & $16 \mathrm{QAM}$ \\
\hline Channel Coding & LDPC, Rate: $2 / 5$ and $5 / 6$ \\
\hline Channel Model & CDL-C (Urban Macro-cell) \\
\hline Number of TX Antennas & 8 \\
\hline Number of RX Antennas & 2 \\
\hline Equalizer & MMSE \\
\hline PDSCH Mapping Type & Singular Value Decomposition
\end{tabular}




\section{A. Classification Performance}

The classification strategy relies on two main features: (1) Log-likelihood ratios $\left(B E R_{L}\right)$ and (2) Channel state estimation (CSI). The distribution of these features with the corresponded decodability outcomes is represented in Fig. 2. It is notable from the samples' behavior that some of the received blocks share the same $B E R_{L}$ feature, although the decodability outcomes are different. We conclude that relying only on $B E R_{L}$ cannot be considered a safe option to obtain an accurate classifier. Therefore, we introduced the new CSI feature that can contribute to increasing the variance between the features, thus providing the classifier with a broader space to distinguish the different outcomes effectively. The accuracy

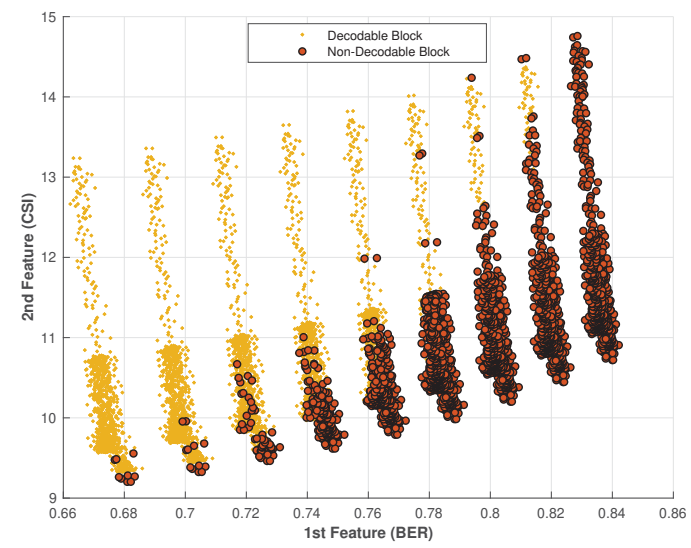

Fig. 2. Distribution of the decodability outcomes among the features.

of the proposed E-HARQ can be evaluated by finding the ratio of a number of correct predictions to the total number of input samples, see (9).

$$
\text { Accuracy }=\frac{\text { CorrectPredictions }}{\text { TotalPredictions }} * 100 .
$$

The classification accuracy, as illustrated in Table IV, reveals about $12 \%$ and $14 \%$ enhancement on the proposed classifier performance in comparison with the other classifiers that relied on just one feature (i.e., ML-based and HT-based, respectively). Further, the robustness of the proposed classifier is evaluated by varying the channel coding rate (CR) to 5/6; it seems that the performance gain is still useful with reasonable degradation on the overall system performance for all the classifiers due to using a higher channel coding rate.

\section{B. System Performance}

The link performance is evaluated first by comparing the BLER for the traditional wireless link and the same link when the E-HARQ feedback mechanism is active (i.e., at various features). In a comparison between the proposed model and another E-HARQ scheme, the simulation results, as shown in Fig. 3, demonstrate the superiority of the proposed model at all SNR regions. This behavior can be attributed to the new feature's ability to efficiently increase the variance between
TABLE IV

CLASSIFICATION COMPARISON BETWEEN DIFFERENT METHODS

\begin{tabular}{|c|c|c|c|}
\cline { 3 - 4 } \multicolumn{2}{c|}{} & \multicolumn{2}{c|}{ Accuracy \% } \\
\hline Method & Feature & CR= 2/5 & CR= 5/6 \\
\hline Hard Threshold (HT) & $B E R_{L}$ & 81 & 79 \\
\hline Logistic Regression (ML) & $B E R_{L}$ & 83 & 80 \\
\hline Logistic Regression (ML) & $B E R_{L}, C S I$ & 95 & 89 \\
\hline
\end{tabular}

the training samples on another scale; this can give the classifier additional flexibility to distinguish the closely spaced outcomes, thus increasing the accuracy in decision-making. Compared to the traditional HARQ, the emerging gap due to the application of E-HARQ narrows in favor of the new proposal, then notably begins to regress at higher SNR values. To explain such behavior, let us find the ratio of the incorrectly

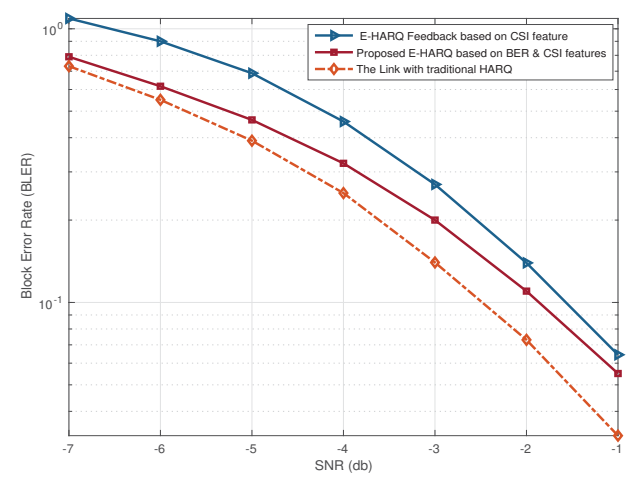

Fig. 3. Block error rate comparison between the proposed E-HARQ (Twofeatures based), E-HARQ (one-features based) and the standard HARQ, Clustered Delay Line channel model, 16 QAM, CR=2/5.

estimated outcomes to the total observations, which refers to the misclassification rate. Since the BLER is not considered at this fraction, this provides a different angle to evaluate the model performance. It is notable, as shown in Fig. 4 that most of the classifiers' performance is significantly varying at these critical SNR regions. This calls for more attention to validate the classifier performance in this particular region. Further, all methods' classifying ability starts to decline and reach a peak at $\mathrm{SNR}=-2.4 \mathrm{~dB}$ for the proposed algorithm and -3.7 for the other classifier. In this particular region, the decodability outcomes of the collected samples at each class are relatively even; and the features' variance becomes significantly close, making it difficult for the classifier to perform the right decision. Notably, the accuracy of all E-HARQ predictors at high and low SNR regions is fairly reliable. This behavior refers to the fact that most of the decodability outcomes at these SNR regions have a monopolistic majority on a one-class among the other. We further show in Fig. 5 that usage of the proposed E-HARQ enhanced the link throughput comparing to another E-HARQ scheme. Notably, the throughput is increased 
(almost) linearly in all cases. The gaps between the E-HARQ classifiers and traditional HARQ start to minimize at high SNR values because the packet is almost always decoded correctly in the first round(s).

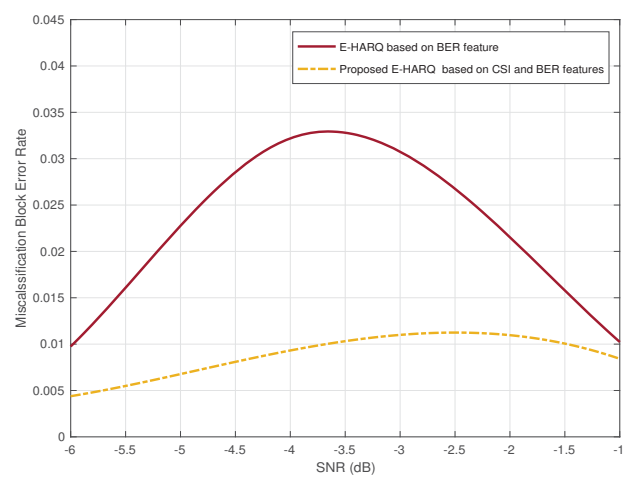

Fig. 4. The Misclassification rate versus the SNR, Clustered Delay Line channel model, 16 QAM, CR=2/5.

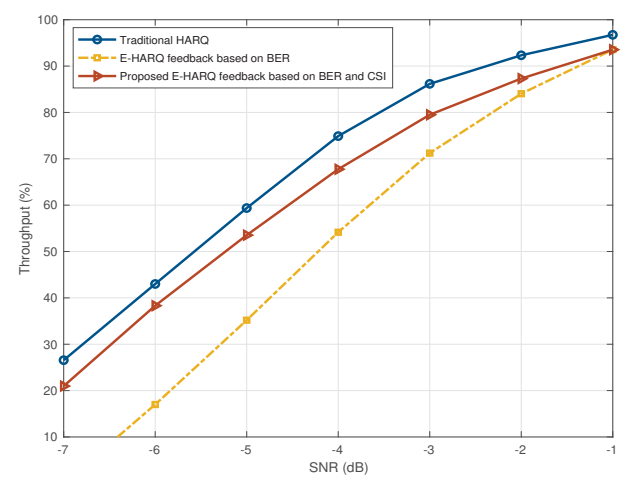

Fig. 5. On the effect of E-HARQ on the link throughput, Clustered Delay Line channel model, 16 QAM, CR=2/5.

To summarize, employing E-HARQ comes with possible misclassification cost because the feedback is estimated based on a noisy coded-signal that has not yet fully benefited from the error correction process (i.e., channel decoding). However, we demonstrated the practical feasibility of introducing the new $C S I$ feature, as the overall performance is improved in terms of BLER, classification accuracy, and the link throughput with faster feedback releasing.

\section{CONCLUSION}

In this paper we proposed and evaluated the performance of an E-HARQ scheme based on ML. The proposed scheme shows the capability of the transmitter to speed up the feedback releasing, compared to other techniques in the relevant literature. Furthermore, simulation results revealed a $12 \%$ enhancement on the prediction accuracy, following the introduction of a new feature that concluded from the channel state estimation. However, the research efforts undertaken into $\mathrm{Ar}$ tificial Intelligence (AI) technology (in particular for URLLC) still remain in their early stages. In order to take advantage of these efforts to the industrial sector, it is vital to consider the capability of the promising AI-based models to learn and analyze the characteristics of actual sophisticated wireless channels. This could be realized by relying on a solid dataset to train and compare the performance of different learning algorithms. However, it should be noted that it is challenging to locate open data sets for wireless communications, due to the protection and privacy regulations in place regarding data sharing. In this study, we aimed to narrow this gap by training the proposed model with a standardized dataset that was collected from a standard-compliant waveform of 3GPP 5 G NR Release 15. Our plans for future work include further investigations of the capability of the ML model to retrain its parameters online, in order to retain its efficiency in the field, even when the characteristics of the real channel don't match the channel models considered during the training stages.

\section{ACKNOWLEDGMENT}

We would like to acknowledge the support of the University of Surrey 5GIC (http://www.surrey.ac.uk/5gic) members for this work.

\section{REFERENCES}

[1] H. Ji, S. Park, J. Yeo, Y. Kim, J. Lee, and B. Shim, "Ultra-reliable and low-latency communications in 5G downlink: Physical layer aspects," IEEE Wireless Communications, vol. 25, no. 3, pp. 124-130, JUNE 2018.

[2] B. Goektepe, S. Faehse, L. Thiele, T. Schierl, and C. Hellge, "Subcodebased early HARQ for 5G," in 2018 IEEE International Conference on Communications Workshops (ICC Workshops). IEEE, 2018, pp. 1-6.

[3] G. Berardinelli, S. R. Khosravirad, K. I. Pedersen, F. Frederiksen, and P. Mogensen, "Enabling early HARQ feedback in 5G networks," in 2016 IEEE 83rd Vehicular Technology Conference (VTC Spring), May 2016, pp. 1-5.

[4] M. Iwabuchi, A. Benjebbour, Y. Kishiyama, and Y. Okumura, "Field experiments on 5G ultra-reliable low-latency communication (URLLC)," NTT DOCOMO Tech. Jour, vol. 20, no. 1, pp. 14-23, 2018.

[5] N. Strodthoff, B. Göktepe, T. Schierl, C. Hellge, and W. Samek, "Enhanced machine learning techniques for early HARQ feedback prediction in 5G," IEEE Journal on Selected Areas in Communications, vol. 37, no. 11, pp. 2573-2587, 2019.

[6] K. I. Pedersen, S. R. Khosravirad, G. Berardinelli, and F. Frederiksen, "Rethink hybrid automatic repeat request design for $5 \mathrm{G}$ : Five configurable enhancements," IEEE Wireless Communications, vol. 24, no. 6, pp. 154-160, 2017.

[7] "Minimum requirements related to technical performance for imt-2020 radio interface(s)," 2017.

[8] H. Holma, A. Toskala, and T. Nakamura, 5G Technology: 3GPP New Radio, 2nd ed. Hoboken, New Jersey: Wiley-Blackwell, 2019.

[9] P. Popovski, C. Stefanović, J. J. Nielsen, E. De Carvalho, M. Angjelichinoski, K. F. Trillingsgaard, and A.-S. Bana, "Wireless access in ultrareliable low-latency communication (URLLC)," IEEE Transactions on Communications, 2019.

[10] 3GPP, "Study on channel model for frequencies from 0.5 to $100 \mathrm{GHz}$," 3GPP TSG RAN, TR 38.901.

[11] S. Coleri, M. Ergen, A. Puri, and A. Bahai, "Channel estimation techniques based on pilot arrangement in OFDM systems," IEEE Transactions on broadcasting, vol. 48, no. 3, pp. 223-229, 2002.

[12] J. Goodman, "Exponential priors for maximum entropy models," in Proceedings of the Human Language Technology Conference of the North American Chapter of the Association for Computational Linguistics: HLT-NAACL 2004, 2004, pp. 305-312.

[13] 3GPP, "Release description; release 15)," 3rd Generation Partnership Project; Technical Specification Group Radio Access Network, TR21.915. 